\title{
The Mathematical Life of Fan Chung
}

Steve Butler

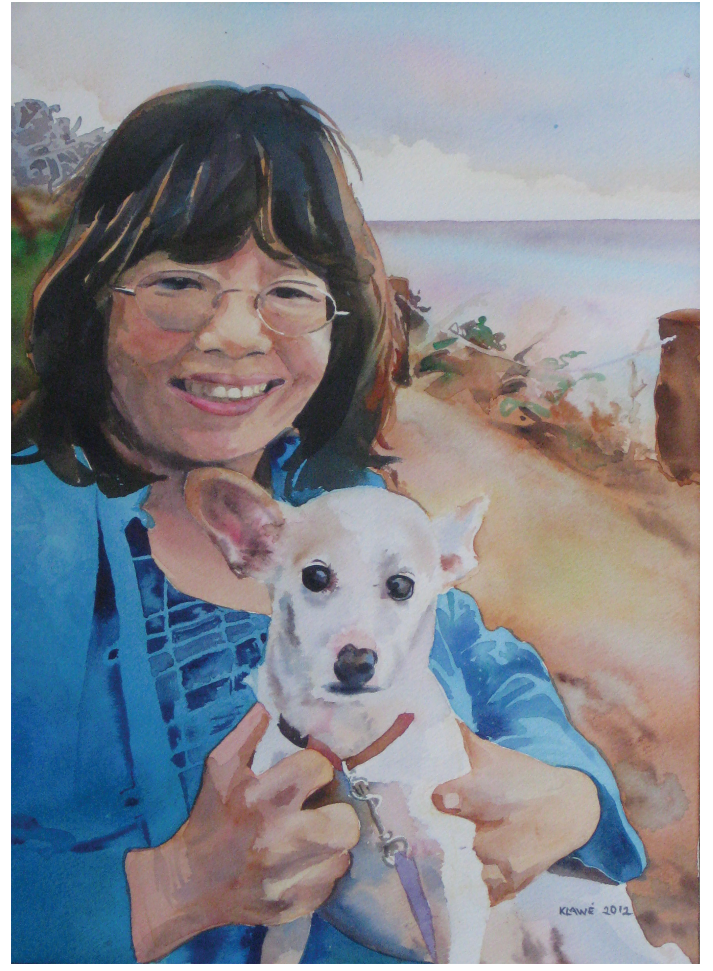

In 1971, Fan Chung was a promising graduate student at the University of Pennsylvania who had earned the highest marks on the qualifying exam. Her performance attracted the attention of a faculty member who sought her out in hopes of recruiting her as a student. He gave her a paper in the area of Ramsey theory to read and an appointment to meet a week later. At that appointment she pointed to a result and declared that she could "do a little better." This was the start of a five-decade (and counting) career in mathematics that has produced three books $[9,11,14]$,

Steve Butler is the Barbara J. Janson Professor of Mathematics at Iowa State University. His email address is but1er@iastate.edu.

Communicated by Notices Associate Editor Emilie Purvine.

For permission to reprint this article, please contact:

reprint-permission@ams.org.

DOI: https://doi.org/10.1090/noti2036 approximately three hundred publications, ${ }^{1}$ as well as countless talks, awards, and honors.

Her research has covered a broad spectrum of topics in combinatorics and graph theory. We will touch on several of these areas, including Ramsey theory, universal structures, spectral graph theory, quasi-random graphs, and general random graph theory. More recently, Fan Chung has been involved with the mathematics of large networks and PageRank. A nice survey about the basic results in this direction has previously appeared in the Notices [8]; we refer the reader to this article for more information on these topics.

Given her prodigious research career, which is still continuing, we will be able to discuss only a part of Fan Chung's mathematical results. One major theme that we will see running through her research is dealing with very large objects. In many cases, our intuition and understanding is well established for smaller objects and objects with high degrees of symmetry. But the very large and more jumbled objects are difficult to understand, and in many cases even describing them requires nontrivial tools.

Most of the topics which we will touch on deal with graph theory. A graph is a collection of objects (nodes or vertices) together with connections between objects (edges). Because of their flexibility, graphs can be used to describe a wide variety of phenomena, from the purely abstract (vertices are elements in a group; edges are produced by some set of generators) to the heavily applied (vertices are webpages; edges correspond to links between webpages). This flexibility makes graph theory a useful tool but also one which can be unwieldy given the great variety of graphs. We will restrict ourselves generally to simple graphs and use standard terminology that can be found in any introductory graph textbook.

\section{Ramsey Theory}

The professor that Fan Chung met with was Herb Wilf (who would go on to become her advisor). The result that she was able to improve upon would lead to her first publication [16] and become a part of her dissertation.

${ }^{1}$ Most of them available at her website http://math. ucsd.edu/ fan/, together with extensive additional materials. 
The basic tenet of Ramsey theory is that if you have a large enough structure, then there must be some small part that is well behaved. As a simple example, let us take the complete graph on six vertices (all possible edges joining six different vertices) and individually color the edges either red or blue. We claim that there is a triangle that is either all red or all blue (by which we mean three vertices where the edges connecting them will be all the same color). The proof proceeds by selecting a vertex and then noting by the pigeonhole principle that at least three edges out of that vertex have the same color, say red. If the triangle formed by the vertices to which the edges connect has any red edge, then a red triangle is formed; if not, then all edges are blue and a blue triangle is formed.

More generally, it is known that for any $n_{1}, \ldots, n_{k}$ there is a finite number, denoted $r:=r\left(n_{1}, \ldots, n_{k}\right)$, so that if the edges of the complete graph on $r$ vertices are colored with $k$ colors, then for some $i$ with $1 \leq i \leq k$ there is a complete graph on $n_{i}$ vertices with all edges of the $i$ th color. This can be established with combinations of the pigeonhole principle and induction arguments. So existence of these numbers can be established with simple tools; the challenge in Ramsey theory comes from determining (or even just bounding) the value of $r$. To show that $r \geq k$, some coloring of the complete graph on $k-1$ must be produced avoiding all possible monochromatic copies of graphs of the appropriate size. To show that $r \leq \ell$, every coloring of the complete graph on $\ell$ vertices must be shown to contain a monochromatic copy of a graph of an appropriate size. The challenge is that the numbers for $r$ tend to grow rapidly, and so the number of possible colorings to consider (for upper or lower bounds) far exceeds a computational search.

The problem with which Fan Chung was presented dealt with $r(3,3,3,3)$. It was previously known that $r(3,3,3)=17$, and she built on this result to show that

$$
r(3,3,3,3) \geq 51 .
$$

This result is still(!) the best-known lower bound (possibly because it is the correct value). By comparison the best-known upper bound is $r(3,3,3,3) \leq 62$ [20]. Her graduate work further explored multicolor Ramsey numbers, and her early career at Bell Labs continued to allow her to work and interact with people working in Ramsey theory. This led to additional work in the field, with an emphasis on constructive lower bounds for Ramsey numbers and generalizations where the complete graphs were replaced with other graphs.

\section{Universal Structures}

After graduate school, Fan Chung immediately started working for Bell Labs (later Bellcore). At the time Bell Labs had a large and vibrant mathematical research center

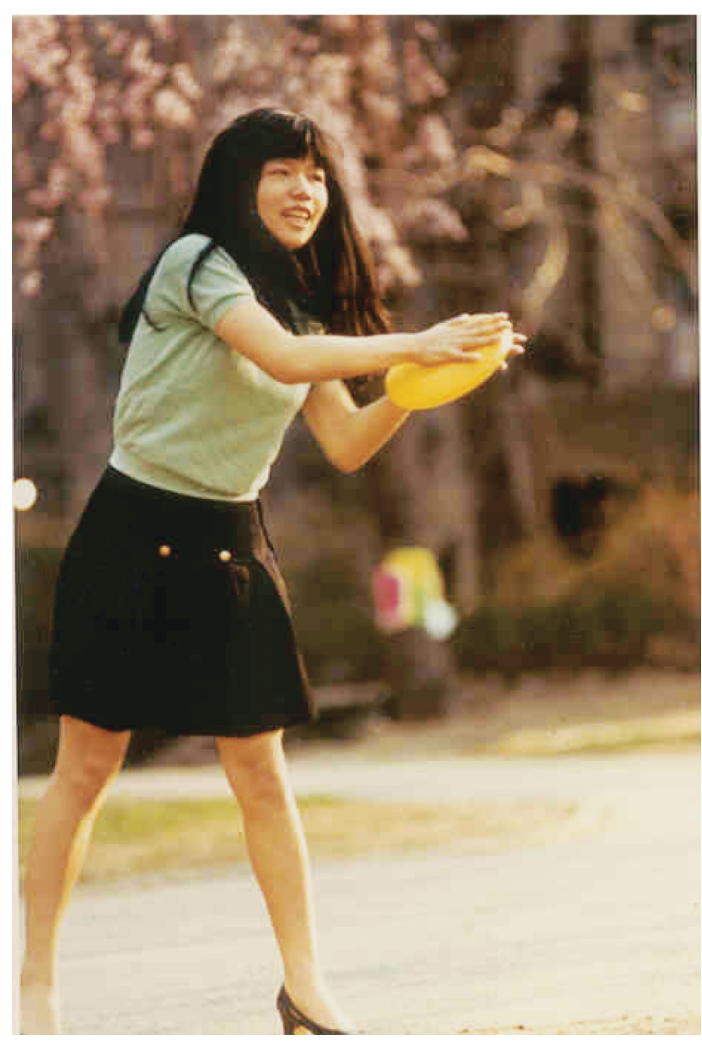

Figure 1. Fan Chung shortly after graduating from the University of Pennsylvania.

under the direction of Henry Pollak. The hallways were lined with offices of mathematicians whose doors were always open. The environment led to a large culture of people sharing problems and doing collaborative work.

At the same time Bell Labs was very generous in giving their scientists sufficient time to engage in basic research. So while Fan Chung would work on problems of direct interest to telecommunications (for example, exploration of Steiner trees that looks at the minimization of connections of points in a space [5] or network robustness [1]), there was also time to explore topics that were more theoretical and less practical.

One of the directions that Fan Chung's research took at this time was into universal structures. Given a fixed family $\mathcal{F}$ of objects, the goal is to create an object that contains all elements of $\mathcal{F}$ somewhere inside. As a simple example, de Bruijn sequences pack all possible 0-1 patterns of length $n$ into a cyclic pattern of length $2^{n}$ where any pattern can be found in some $n$ consecutive terms (possibly with wrap around). For example 0000111101100101 contains all binary patterns of length 4 as consecutive elements.

Fan Chung and her collaborators were primarily interested in the case when $\mathcal{F}$ consists of families of graphs. Existence of these structures is usually straightforward; for example, a universal graph for a fixed family of graphs can be found by taking the disjoint union of all the graphs in 


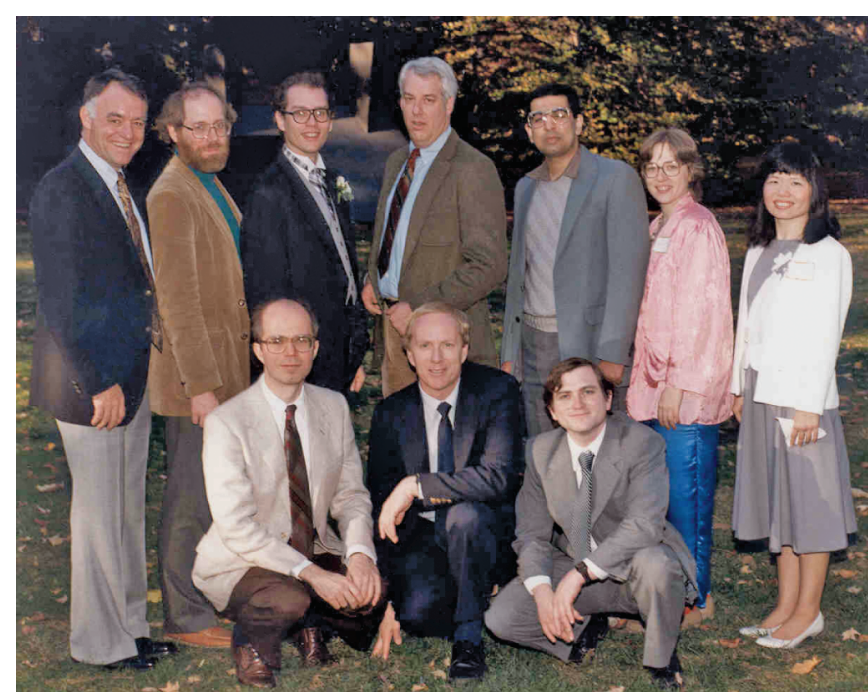

Figure 2. Some members of the Mathematical Foundation of Computing Group at Bell Labs. From the left, the back row Ed Coffman, David Johnson, Peter Shor, Mike Garey, Pravin Vaidya, Karen Collins, Fan Chung; and the front row - Andrew Odlyzko, Ron Graham, Robert Wilber.

the family. Additional constraints might be placed on the structure; for example, you might want to have a tree that contains all smaller trees of a certain size, but even in these cases existence is usually straightforward. As an example of a universal object involving graphs, the tree in Figure 3 contains all trees with 8 vertices as a subtree.

The challenge is to construct small universal graphs, and Fan Chung and her collaborators were able to do this for sparse graphs, planar graphs, and graphs with bounded maximum degree. They also considered the problem when the graphs were required to be induced [13].

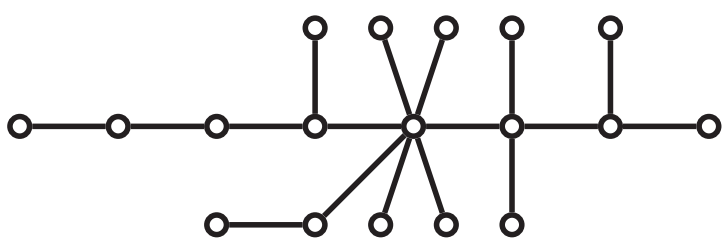

Figure 3. A tree on 18 vertices which contains all trees on 8 vertices as a subtree.

During her time at Bell Labs and Bellcore, Fan Chung had the opportunity to work with Paul Erdős on many occasions, and Erdős was a frequent guest at her home. Erdős was a unique mathematician (and house guest), and Fan Chung described the experience of collaboration with Erdős in the following way:

Working with Paul was like taking a walk in the hills. Every time when I thought that we had achieved our goal and deserved a rest, Paul pointed to the top of another hill and off we would go. [9]
Fan Chung and Paul Erdős worked on fourteen papers together. ${ }^{2}$ Among these was the topic of unavoidable graphs [3], which in some sense is the complementary problem to universal graphs. Now instead of looking for a large graph that contains some set of small graphs, we find small graphs that must be contained in every large graph.

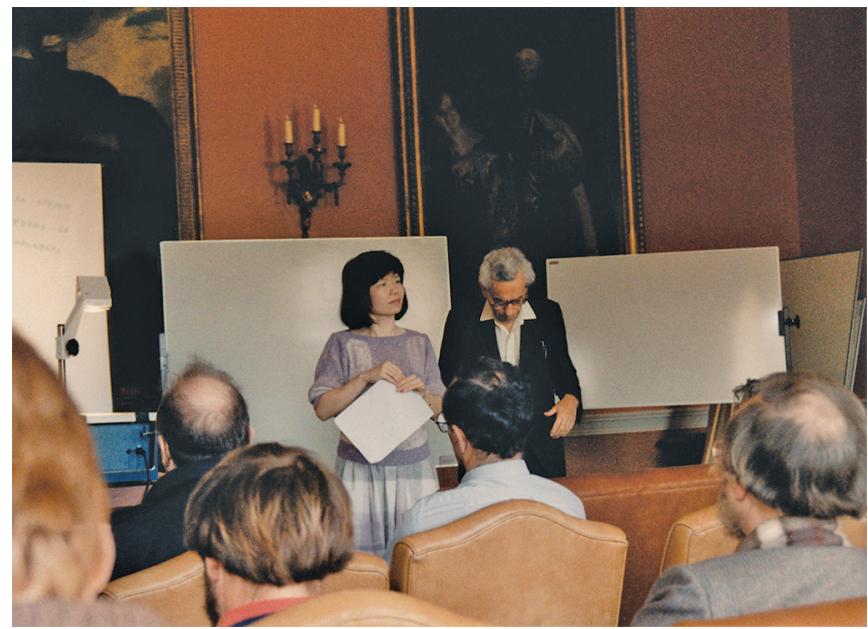

Figure 4. Fan Chung and Paul Erdős at a conference in Cambridge in 1988.

\section{Spectral Graph Theory}

In 1991, while at Bellcore, Fan Chung was able to arrange for the equivalent of a sabbatical at Harvard University. Given that there was not a strong combinatorics presence at the time, this was not the most obvious decision. But upon arriving she soon formed many collaborations, including Shlomo Sternberg (collaborated on the Buckyball), Persi Diaconis (collaborated on random walks), David Mumford (collaborated on planar graphs), and Shing-Tung Yau. Her collaboration with Shing-Tung Yau would grow to include fifteen papers about spectral graph theory (some of this collaborative work can be found in her book on spectral graph theory [14]).

The idea of spectral graph theory is to take a graph and associate it to a matrix with rows and columns indexed by the vertices. From the matrix we then find the eigenvalues, or spectrum, of the graph. So given the graph, we can find the spectrum, and the question becomes: From the spectrum what can we learn about the graph? An apt analogy is how we are able to learn the chemical composition of a distant star from its light spectrum. Of course, there is significant information loss, and so only in a few cases can we explicitly reconstruct the graph. Nevertheless, we

\footnotetext{
${ }^{2}$ Another of their joint papers was published under the pseudonym "G. W. Peck" [19], a collaboration of six mathematicians where each author involved was given a letter. This is sometimes (jokingly) referred to in Erdôs collaboration circles as an imaginary coauthorship. Including this paper brings the count to $14+i$.
} 
are able to learn some useful information about the graph from the spectrum.

A key decision in spectral graph theory is selecting the matrix to be used. The most common matrices are the adjacency matrix, denoted $A$ (where $A_{u, v}$ is 1 if the vertices $u$ and $v$ are adjacent and 0 otherwise), or the Laplacian matrix, denoted $L$ (with $L=D-A$, where $A$ is the adjacency matrix and $D$ is a diagonal matrix of degrees). Different matrices are better at identifying different behavior in the graph, so there is not a single best matrix.

Before going to Harvard University, Fan Chung had already worked in spectral graph theory. Some of her earlier work at Bell Labs focused on expander graphs, which are basic building blocks for robust communication networks. With Noga Alon she had established a connection between the eigenvalues of the adjacency matrix of a regular graph and the placement of edges.

Lemma 1 (Expander Mixing Lemma (regular graphs) ([1])). Let $e(S, T)$ be the number of edges with one endpoint in $S$ and one endpoint in $T$ (the number of edges between $S$ and $T$; by convention edges in $S \cap T$ are double counted). Let $G$ be a d-regular graph with eigenvalues of the adjacency matrix $\alpha_{n} \leq \cdots \leq \alpha_{2} \leq \alpha_{1}=d$ and let $\alpha=\max \left\{\left|\alpha_{n}\right|,\left|\alpha_{2}\right|\right\}$. Then for all $S, T \subseteq V$,

$$
\left|e(S, T)-\frac{d}{n}\right| S|| T|| \leq \alpha \sqrt{|S||T|} .
$$

The left-hand side of (1) looks at the difference between the number of edges between $S$ and $T$ and the expected number of edges if they had been placed "randomly." The right-hand side indicates that this difference is bounded by how well the nontrivial eigenvalues cluster around 0 .

The matrix that Fan Chung and Shing-Tung Yau decided to look at was termed the normalized Laplacian, denoted $\mathcal{L}$. If we let $\operatorname{deg}(u)$ denote the degree of a vertex $u$, then this matrix is defined entrywise by

$$
\mathcal{L}_{u, v}= \begin{cases}1 & \text { if } u=v \text { and } \operatorname{deg}(u)>0 \\ -\frac{1}{\sqrt{\operatorname{deg}(u) \operatorname{deg}(v)}} & \text { if } u \text { and } v \text { are adjacent } \\ 0 & \text { otherwise. }\end{cases}
$$

When there are no isolated vertices this simplifies to $\mathcal{L}=$ $D^{-1 / 2} L D^{-1 / 2}$. The motivation for this variation was that it mirrors what happens in the continuous setting with the Laplacian operator on a manifold and allows for a (semi)clean translation of results from the continuous to the discrete. Examples include Harnack inequalities, Sobolev inequalities, and the Cheeger inequalities.

For a subset of vertices, $S \subseteq V$, let the volume of the subset be given by $\operatorname{vol}(S)=\sum_{v \in S} \operatorname{deg}(v)$. (Roughly speaking, the volume is a measurement of the size of a subset of vertices but now with emphasis placed on the edges.) The
Cheeger constant for a connected graph is defined to be

$$
h(G)=\min _{\emptyset \subset S \subset V} \frac{e(S, V \backslash S)}{\min \{\operatorname{vol}(S), \operatorname{vol}(V \backslash S)\}} .
$$

When there are a few edges that split the graph into two large parts, then $h(G)$ is small, so roughly speaking the smaller the value of $h(G)$ the more poorly connected the graph. We have the following.

Theorem 1 (Cheeger inequality (Chung [14])). Let $0=$ $\lambda_{0}<\lambda_{1} \leq \cdots \leq \lambda_{n-1}$ be the eigenvalues of the normalized Laplacian for a connected graph $G$. Then

$$
\frac{1}{2}(h(G))^{2}<\lambda_{1} \leq 2 h(G) .
$$

Moreover, up to a constant the upper and lower bounds cannot be improved.

This shows that the existence of a bottleneck in the graph is heavily tied to the size of the first nontrivial eigenvalue of the normalized Laplacian.

The normalized Laplacian can be formed by taking the probability transition matrix of a random walk $\left(D^{-1} A\right)$, symmetrizing it $\left(D^{-1 / 2} A D^{-1 / 2}\right)$, and then negating and shifting the eigenvalues $\left(\mathcal{L}=I-D^{-1 / 2} A D^{-1 / 2}\right)$. As a consequence there is a close connection to random walks on graphs and the normalized Laplacian. Fan Chung has strongly utilized this connection in her work on the normalized Laplacian.

From a combinatorial perspective the normalized Laplacian is not an obvious matrix to choose. The individual entries of the matrix are unwieldy, but even more problematic is that the eigenvalues tend to be poor at producing quantitative measurements of the graph. As an example, it is known that the number of edges cannot always be determined from the spectrum of the normalized Laplacian of the graph. However, the normalized Laplacian tends to be strong at qualitative measurements such as diffusion and clustering. Through the work of Fan Chung the normalized Laplacian has been gaining traction in the spectral graph theory community, especially for applications in optimization and computer vision.

One way to view what is happening with the normalization is that the importance of a vertex is now based on how connected that vertex is to the rest of the graph. This perspective change is what is needed when dealing with nonregular graphs. For example, the Expander Mixing Lemma was originally stated for regular graphs, but by transitioning to the normalized Laplacian and changing the perspective to connections (so for a subset of vertices the measure is the sum of the degrees which is the volume) we have the following.

Lemma 2 (Expander mixing (general graphs) ([14])). Let $e(S, T)$ be the number of edges with one endpoint in $S$ and one endpoint in $T$ (the number of edges between $S$ and $T$; by 
convention edges in $S \cap T$ are double counted). Let $G$ be a graph with eigenvalues of the normalized Laplacian matrix $0=\lambda_{0} \leq$ $\lambda_{1} \leq \cdots \leq \lambda_{n-1}$ and let $\lambda=\max \left\{\left|1-\lambda_{1}\right|,\left|1-\lambda_{n-1}\right|\right\}$. Then for all $S, T \subseteq V$,

$$
\left|e(S, T)-\frac{\operatorname{vol}(S) \operatorname{vol}(T)}{\operatorname{vol}(V)}\right| \leq \alpha \sqrt{\operatorname{vol}(S) \operatorname{vol}(T)} .
$$

Fan Chung has become a champion of spectral graph theory and more particularly for the normalized Laplacian. Her efforts in the field permeate through her research and her collaborations (many of her academic descendants continue to use the tools of spectral graph theory in their research).

\section{Quasi-random Graphs}

Looking at either variation of the Expander Mixing Lemma ((1) or (2)) we can translate it roughly as "if the nontrivial eigenvalues of the adjacency (or normalized Laplacian) matrix are close to 0 (or 1 ), then the edges of the graph are behaving fairly randomly with respect to their placement." So we can think of the eigenvalues as giving a measurement of how well a graph behaves like a random graph.

In general, we know that random graphs (graphs generated by randomly deciding to include each individual edge with some specified probability) are well behaved and have nice properties. We even know that in some sense almost all graphs behave like a random graph. The challenge is how do we know if a particular graph will behave like a random graph (in general graphs generated by some deterministic properties tend to have more structure than a random graph would exhibit). ${ }^{3}$

Let $q=4 k+1$ be a prime power. We can construct the Paley graph on $q$ vertices where the vertices are the residue classes modulo $q$ and two residue classes are connected if and only if their difference is a quadratic residue (so $a$ and $b$ are joined by an edge if and only if $a-b \equiv t^{2}(\bmod q)$ for some $t$ ). The Paley graph on 17 vertices is shown in Figure 5. Does this graph behave like a random graph? (It certainly does not look random, and the rules for forming it involve a substantial amount of symmetry.)

Fan Chung, Ron Graham, and Richard Wilson [7] introduced the notion of quasi-random graphs or, more appropriately, quasi-random graph properties. These are a set of graph properties that have the following two important characteristics: (1) the properties are satisfied by a random graph, and (2) if one property is satisfied up to some small error, then any other property is satisfied up to some related small error where the errors both go to 0 together.

The original list of quasi-random graph properties included the following (it has expanded over time and

\footnotetext{
${ }^{3}$ This problem is similar to trying to find a needle in a needlestack, only to reach in and discover that we had grabbed a piece of hay.
}

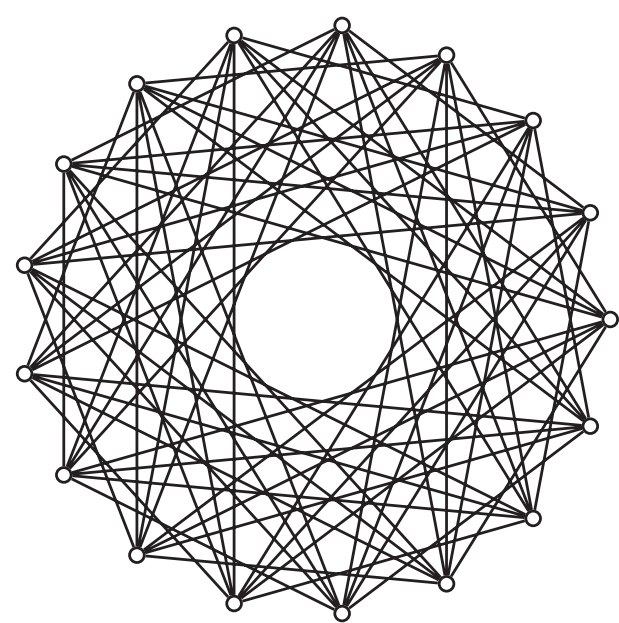

Figure 5. The Paley graph on 17 vertices where vertices are residue classes modulo 17 and two residue classes are connected if their difference belongs to $\{ \pm 1, \pm 2, \pm 4, \pm 8\}$.

as more researchers have discovered the power of quasirandom graphs).

1. Let $k \geq 4$ be fixed. The number of copies of any graph on $k$ vertices present in the graph as a labeled induced

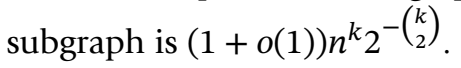

2. The number of edges present in the graph is at least $\left(\frac{1}{4}+o(1)\right) n^{2}$, and the number of four-cycles is at most $\left(\frac{1}{16}+o(1)\right) n^{4}$.

3. Let $\alpha_{n} \leq \cdots \leq \alpha_{1}$ be the eigenvalues of the adjacency matrix. The number of edges in the graph is at least $\left(\frac{1}{4}+o(1)\right) n^{2}, \alpha_{1}=\left(\frac{1}{2}+o(1)\right) n, \alpha_{2}=o(n)$.

4. For any $S \subseteq V$, we have $e(S, S)=\frac{1}{2}|S|^{2}+o\left(n^{2}\right)$.

The power comes from noting that some of these properties are easy to verify and others are useful to apply. For example, the Paley graphs on $q$ vertices can be shown to satisfy the second property on the list, and therefore they satisfy them all. In particular, any small graph shows up as an induced subgraph roughly the expected number of times as the size of the Paley graph grows large. As with random graphs, most graphs are quasi-random, but now we have relatively small computational checks that can establish being quasi-random.

Fan Chung and Ron Graham have been able to extend the notions of quasi-randomness to many additional topics, including hypergraphs, sparse graphs, graphs with expected degree sequences, subsets of $\mathbb{Z}_{n}$, tournaments, and more. $^{4}$

\footnotetext{
${ }^{4}$ Fan and Ron were so happy with the quasi-random results that at one point they had a vehicle with the license plate that read QRANDOM. Fan's current license plate reads THEOREM.
} 


\section{General Random Graph Theory}

In 1995, Fan Chung transitioned from industry into academia, taking up a position at the University of Pennsylvania in both the mathematics and computer science departments. A few years later she would move to UC San Diego, where she remains to this day. As she entered into academia, her research took on a new dimension as she delved into the mathematics of complex networks and random graph models.

The most well-known and studied random graph model is based on the work of Erdős and Rényi. In these graphs we start with $n$ vertices, and for each edge we include it with probability $p$ (e.g., by flipping a weighted coin). These graphs have many wonderful properties, and many books have been written on the subject.

One of the properties in the Erdős-Rényi model is that the random graphs tend to be almost regular (all degrees are roughly equal). This is an issue, because the graphs that have arisen in network data (such as phone networks and the internet graph), biological data, and social networks do not exhibit this regular behavior. At the same time, given that they are formed from disparate processes, they seem to share similar structures. For example, they tend to be globally sparse (not many more edges than there are vertices) and also exhibit the "small world phenomenon" (between any two vertices there is a short path). In terms of the degree sequences, these tend to follow a power law distribution; namely, the number of vertices of degree $k$ is about $k^{-\beta}$ for some $\beta$.

To address this issue, Fan Chung worked with her student Linyuan (Lincoln) Lu to rigorously introduce a random graph model with a given degree sequence. The basic idea is that instead of including each edge with the same probability, we first assign a weight to each vertex, $w_{u}$ (interpreted as the expected degree), and the probability of adding an edge is proportional to $w_{u} w_{v}$ (so vertices with high degrees are more likely connected, while vertices with low degrees are less likely connected). Through careful analysis they were able to analyze issues about connectivity, the sizes of the largest components, spectra for various matrices, and more [11]. This model has since been widely used in the community of network sciences and is a better tool for studying power law graphs.

Fan Chung saw the importance of the rigorous mathematical analysis of large complex networks and so in 2003 founded the journal Internet Mathematics.

\section{Additional Contributions}

While we have hit on some of the larger themes of Fan Chung's work, there is still a significant number of topics that have not yet been mentioned, including algebraic combinatorics, posets, combinatorial number theory, discrete geometry, bin packing, and even the mathematics of juggling (she holds the rare distinction of having written more papers about the mathematics of juggling than the number of balls she is able to juggle). These papers span the length of her career and include some beautiful gems of mathematics waiting for someone to pick them up and push the results further.

6.1. Happy ending problem. In 1935 Paul Erdős and George Szekeres [17] considered the problem of forming convex hulls from a set of given points in the plane. In particular, they showed that for any given $k$ if there are sufficiently many points in general position (no three on a line), then some $k$ of the points must form a convex $k$ gon. ${ }^{5}$

More precisely, if we let $f(k)$ denote the minimum number of points needed to guarantee that any $f(k)$ points contain a convex $k$-gon, then Erdős and Szekeres showed that

$$
2^{n-2}+1 \leq f(k) \leq\left(\begin{array}{c}
2 n-4 \\
n-2
\end{array}\right)+1 .
$$

It was widely believed that the lower bound was the correct answer. However, the upper bound, which has size on the order of $4^{n}$, stubbornly refused to budge for over sixty years. Fan Chung and Ron Graham [6] decided to revisit this problem and were able to make the first progress on the bounds of $f(k)$ since the problem had appeared; they got rid of the " +1 " in the upper bound by exploiting some symmetry. While this was a modest improvement, it soon set off a flurry of activity in the community, and the upper bound continued to drop. Recently Andrew Suk [21] made a breakthrough in the problem and was able to show that

$$
f(k)=2^{(1+o(1)) n} .
$$

This is one of several examples where Fan Chung was able to help keep improving existing bounds on wellknown problems (others include the minimum number of distinct distances for $n$ points in the plane $[2,15]$ and the hypergraph Turan density problem [10]).

It should be noted that Fan Chung and Ron Graham, who have been married since 1983, have had an extensive collaboration career with roughly one hundred joint papers. Almost every time that they travel together they pick a problem to work on, showing the old adage that for a mathematician a vacation is a period of uninterrupted work. ${ }^{6}$

6.2. Pebbling. For any (connected) graph $G$ we can play a solitaire-like game on the graph that involves moving pebbles (tokens) between vertices, starting from some initial distribution of pebbles in the graph. The "pebbling" moves work by taking two pebbles from some vertex and then adding one pebble to an adjacent vertex (so that there

\footnotetext{
5 "The happy ending" coming from this work was the marriage of George Szekeres to Esther Klein, who had initially posed the problem.

${ }^{6}$ We wish them many happy years of traveling in the future.
} 


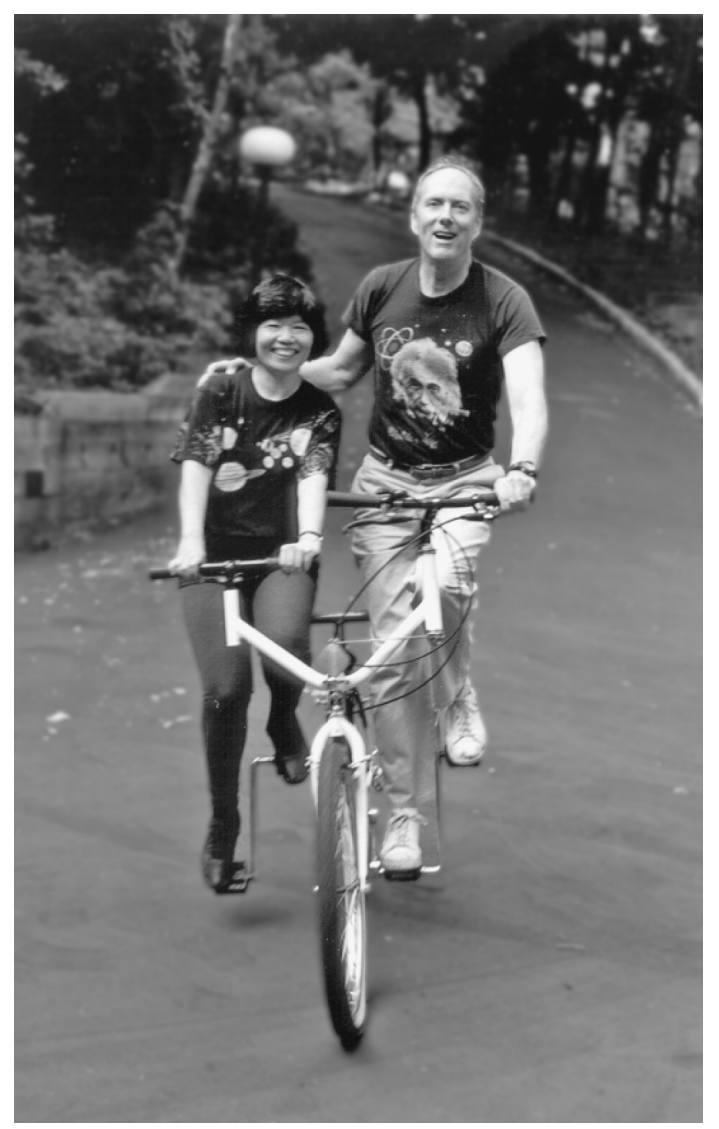

Figure 6. Fan Chung and Ron Graham working on a different type of collaboration.

is a cost of one pebble to move a pebble to an adjacent vertex). The question becomes, what is the fewest number of pebbles needed, denoted $p(G)$, to guarantee that no matter how the pebbles are initially distributed among the vertices, there is always a way to conduct a series of pebbling moves to get at least one pebble to a specified vertex? As an example, in Figure 7 there are eight pebbles spread among the vertices of a 3-cube (the number in the vertex is the current number of pebbles; unlabeled vertices have 0 pebbles). Is it possible to get one pebble to the vertex in yellow using pebbling moves?

Fan Chung [12] showed that if the graph $G$ is the $n$-cube, then $p(G)=2^{n}$. This is best possible, since in general we need at least the number of vertices. Otherwise, if we place a single pebble at every vertex other than the specified vertex, then we cannot do a pebble move. The extreme cases for the $n$-cube are easily verified. If each vertex gets a pebble, then it is trivial; if one vertex gets all the pebbles, then since the diameter is $n$ and we can move the pebbles en masse, losing half the pebbles at each move, we can get to any specified vertex. Of course the more interesting cases are when there is something in between, which she was able to establish by a careful induction argument.

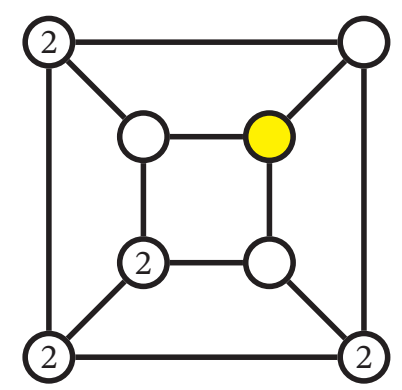

Figure 7. In pebbling we move pebbles around the graph by selecting a vertex with two or more pebbles, removing two pebbles from that vertex, and then adding a pebble at an adjacent vertex. The configuration shown here has eight pebbles initially placed, and the goal is to get one pebble to the yellow vertex.

The problem that the paper was based on was passed on to her by Paul Erdős, who heard the problem from Jeffrey Lagarias and Michael Saks. This is reflective of the culture of combinatorics of sharing problems (heavily influenced by Paul Erdős). She passed the result and paper on to Joe Gallian, who took it to his REU, and there has now grown to be an entire pebbling community with hundreds of papers and countless numbers of student projects involved with pebbling on graphs.

One of the biggest open problems in the pebbling community originated in Fan Chung's paper [12] and is known as Graham's conjecture: Show that $p(G \square H) \leq p(G) p(H)$, where $G \square H$ is the Cartesian product of $G$ and $H$. Given the recent success of the combinatorics community to find the answers to long-standing open problems, perhaps we will soon know the answer to Graham's conjecture.

6.3. Boolean functions. The pebbling problem is only one of many different problems on the $n$-cube that Fan Chung has worked on over the years. This is because of her extensive work in computer science that ties in to Boolean functions.

We can associate the $2^{n}$ vertices of the $n$-cube with $\{0,1\}^{n}$ (or 0-1 strings of length $n$ ) with edges joining strings that differ in a single entry. A function $F:\{0,1\}^{n} \rightarrow\{0,1\}$ is called a Boolean function and can be associated with dividing the vertices of the hypercubes into two disjoint sets $\left(F^{-1}(0)\right.$ and $\left.F^{-1}(1)\right)$.

Suppose that $\left|F^{-1}(1)\right|>2^{n-1}$ and let us consider the induced subgraph of the $n$-cube on the vertices of $F^{-1}(1)$; call it $H$. Fan Chung et al. [4] showed that there must be some vertex $u \in V(H)$ with

$$
\operatorname{deg}_{H}(u)>\frac{1}{2} \log n-\frac{1}{2} \log \log n .
$$

It is possible to have $\left|F^{-1}(1)\right|=2^{n-1}$ and have the graph $H$ consist of isolated vertices (for example, pick all vertices with an odd number of 1's). So this shows that if we add even one more vertex, our degree must go significantly up. 
It was believed that a stronger result was possible, and this was recently established by Hao Huang [18], who was able to show that there must be some vertex with degree at least $\operatorname{deg}_{H}(u) \geq \sqrt{n}$; this result is known as the sensitivity conjecture. This is best possible, as Fan Chung et al. [4] showed that there exists a subset of the $n$-cube of size $2^{n-1}+1$ where $\operatorname{deg}_{H}(u) \leq \sqrt{n}+1$ for all vertices in the corresponding graph of $H$.

\section{Final Thoughts}

The ordering of the major themes we presented is semichronological in terms of how the mathematical work of Fan Chung developed. In the early days, her ability as a strong problem solver was on display as she would solve problems posed by others; as time went on, she started to become a theory builder and became the one laying the foundations of an area to help others to explore.

Fan Chung's work is also highly collaborative. She has said that "Coauthorship is a closer relationship than friendship." Many of her collaborations have turned into long and deep friendships. (After all, collaboration "is more fun.")

Fan Chung's mathematical experiences have made her a highly versatile mathematician with an ability to understand and command an overview of the current state and culture of mathematics. She has used these experiences to mentor scores of colleagues and students, whom she cares about greatly. Many of us who have worked with Fan have benefited from her discerning taste in problems, her ability to bring the right people and opportunities together, and even her occasional reprimand.

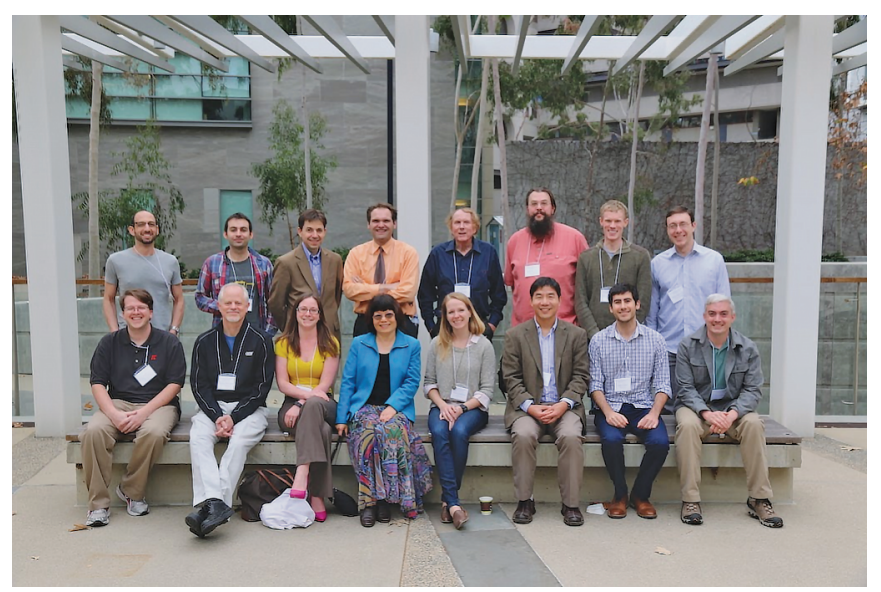

Figure 8. Fan Chung, Ron Graham, and many of their former PhD students (including ten of Fan Chung's seventeen (and counting) students). Taken at the conference Networked Life in January 2016.

Given all of her mathematical output it should also be noted that Fan Chung still finds time to engage in numerous activities outside of mathematics. Currently she finds time to paint, plays the guzheng, performs traditional Chinese dance, and likes working in her yard. Fan Chung serves as a role model, to mathematicians of all types, for what it means to be a successful mathematician.

ACKNOWLEDGMENT. The author thanks Fan Chung for help in preparing the article, as well as the many years of mentoring and friendship.

\section{References}

[1] N. Alon and F. R. K. Chung, Explicit construction of linear sized tolerant networks, Proceedings of the First Japan Conference on Graph Theory and Applications (Hakone, 1986), Discrete Math. 72 (1988), no. 1-3, 15-19, DOI 10.1016/0012-365X(88)90189-6 MR975519

[2] F. R. K. Chung, The number of different distances determined by $n$ points in the plane, J. Combin. Theory Ser. A 36 (1984), no. 3, 342-354, DOI 10.1016/00973165(84)90041-4 MR744082

[3] F. R. K. Chung and P. Erdős, On unavoidable graphs, Combinatorica 3 (1983), no. 2, 167-176, DOI 10.1007/BF02579290. MR726454

[4] F. R. K. Chung, Zoltán Füredi, R. L. Graham, and P. Seymour, On induced subgraphs of the cube, J. Combin. Theory Ser. A 49 (1988), no. 1, 180-187, DOI 10.1016/00973165(88)90034-9 MR957216

[5] F. R. K. Chung and R. L. Graham, On Steiner trees for bounded point sets, Geom. Dedicata 11 (1981), no. 3, 353361, DOI 10.1007/BF00149359 MR627537

[6] F. R. K. Chung and R. L. Graham, Forced convex ngons in the plane, Discrete Comput. Geom. 19 (1998), no. 3, Special Issue, 367-371, DOI 10.1007/PL00009353. MR1608877

[7] F. R. K. Chung, R. L. Graham, and R. M. Wilson, Quasirandom graphs, Combinatorica 9 (1989), no. 4, 345-362, DOI 10.1007/BF02125347. MR1054011

[8] Fan Chung, Graph theory in the information age, Notices Amer. Math. Soc. 57 (2010), no. 6, 726-732. MR2674816

[9] Fan Chung and Ron Graham, Erdős on graphs: His legacy of unsolved problems, A K Peters, Ltd., Wellesley, MA, 1998. MR1601954

[10] Fan Chung and Linyuan Lu, An upper bound for the Turán number $t_{3}(n, 4)$, J. Combin. Theory Ser. A 87 (1999), no. 2, 381-389, DOI 10.1006/jcta.1998.2961. MR1704268

[11] Fan Chung and Linyuan Lu, Complex graphs and networks, CBMS Regional Conference Series in Mathematics, vol. 107, Published for the Conference Board of the Mathematical Sciences, Washington, DC; by the American Mathematical Society, Providence, RI, 2006. MR2248695

[12] Fan R. K. Chung, Pebbling in hypercubes, SIAM J. Discrete Math. 2 (1989), no. 4, 467-472, DOI 10.1137/0402041. MR1018531

[13] Fan R. K. Chung, Universal graphs and induced-universal graphs, J. Graph Theory 14 (1990), no. 4, 443-454, DOI 10.1002/jgt.3190140408 MR1067239 
[14] Fan R. K. Chung, Spectral graph theory, CBMS Regional Conference Series in Mathematics, vol. 92, Published for the Conference Board of the Mathematical Sciences, Washington, DC; by the American Mathematical Society, Providence, RI, 1997. MR1421568

[15] Fan R. K. Chung, E. Szemerédi, and W. T. Trotter, The number of different distances determined by a set of points in the Euclidean plane, Discrete Comput. Geom. 7 (1992), no. 1, 1-11, DOI 10.1007/BF02187820 MR1134448

[16] Fan Rong K. Chung, On the Ramsey numbers $N(3,3, \ldots, 3 ; 2)$, Discrete Math. 5 (1973), 317-321, DOI 10.1016/0012-365X(73)90125-8. MR316290

[17] P. Erdös and G. Szekeres, A combinatorial problem in geometry, Compositio Math. 2 (1935), 463-470. MR1556929

[18] Hao Huang, Induced subgraphs of hypercubes and a proof of the Sensitivity Conjecture, Ann. of Math. (2) 190 (2019), no. 3, 949-955, DOI 10.4007/annals.2019.190.3.6 MR4024566

[19] G. W. Peck, Maximum antichains of rectangular arrays, J. Combin. Theory Ser. A 27 (1979), no. 3, 397-400, DOI 10.1016/0097-3165(79)90035-9. MR555816

[20] Stanisław P. Radziszowski, Small Ramsey numbers, Electron. J. Combin. 1 (1994), Dynamic Survey 1, 30 pp. MR1670625

[21] Andrew Suk, On the Erdós-Szekeres convex polygon problem, J. Amer. Math. Soc. 30 (2017), no. 4, 1047-1053, DOI 10.1090/jams/869 MR3671936

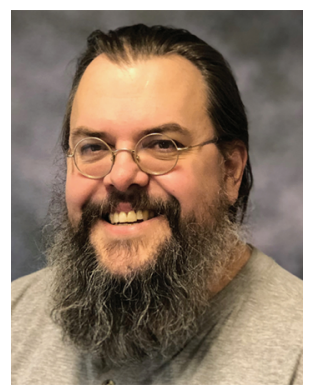

Steve Butler

\section{Credits}

Opening image painted by Maria Klawe.

Figures 1, 2, 4, and 6 are courtesy of Fan Chung.

Figures 3, 5, and 7 are by the author.

Figure 8 is courtesy of Todd Kemp.

Author photo is by Alisha Carroll.

AMS

AUTHOR

RESOURCE

CENTER

The Author Resource Center is a collection of information and tools available to assist you to successfully write, edit, illustrate, and publish your mathematical works.

\section{To begin utilizing these important resources, visit:}

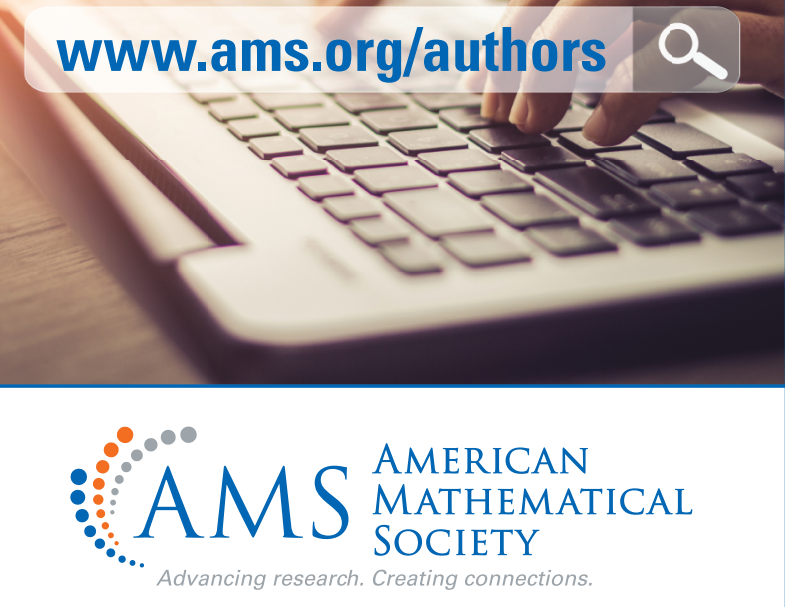

